RECEIVED

JAN 261998

OSTI GA-A22718 CONF-971065 --

\title{
RECENT IMPROVEMENTS TO THE DIII-D NEUTRAL BEAM INSTRUMENTATION AND CONTROL SYSTEM
}

\author{
by \\ D.H. KELLMAN and R. HONG
}

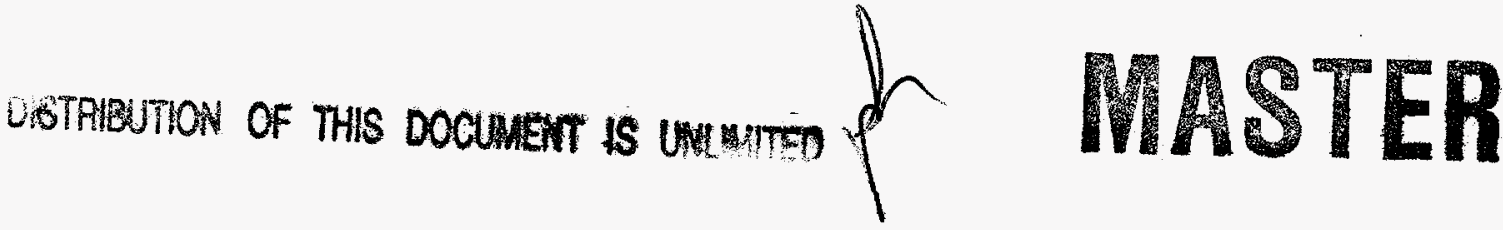




\section{DISCLAIMER}

This report was prepared as an account of work sponsored by an agency of the United States Government. Neither the United States Government nor any agency thereof, nor any of their employees, make any warranty, express or implied, or assumes any legal liability or responsibility for the accuracy, completeness, or usefulness of any information, apparatus, product, or process disclosed, or represents that its use would not infringe privately owned rights. Reference herein to any specific commercial product, process, or service by trade name, trademark, manufacturer, or otherwise does not necessarily constitute or imply its endorsement, recommendation, or favoring by the United States Government or any agency thereof. The views and opinions of authors expressed herein do not necessarily state or reflect those of the United States Government or any agency thereof. 


\section{DISCLAIMER}

Portions of this document may be illegible electronic image products. Images are produced from the best available original document. 


\title{
RECENT IMPROVEMENTS TO THE DIII-D NEUTRAL BEAM INSTRUMENTATION AND CONTROL SYSTEM
}

\author{
by \\ D.H. KELLMAN and R. HONG
}

This is a preprint of a paper to be presented at the 17th IEEE/NPSS Symposium on Fusion Engineering, October 6-11, 1997, San Diego, California and to be published in the Proceedings.

\author{
Work supported by \\ the U.S. Department of Energy \\ under Contract No. DE-AC03-89ER51114
}




\title{
Recent Improvements to the DIII-D Neutral Beam Instrumentation and Control System*
}

\author{
D.H. Kellman and R. Hong \\ General Atomics \\ P.O. Box 85608 , San Diego, California 92186-9784
}

\begin{abstract}
The DIII-D neutral beam (NB) instrumentation and control (I\&C) system provides for operational control and synchronization of the eight DIII-D neutral beam injection systems, as well as for pertinent data acquisition and safety interlocking. Portions of the system have been in use for as many as eighteen years. Over this time, equipment has aged, available technology has improved, and experimental requirements have changed. Recently, improvements were made to the $I \& C$ system to address these issues. With the replacement of the NB control computers, new signal interfacing was required to accommodate the elimination of physical operator panels, in favor of graphical user interface control pages on computer terminal screens. The program in the mode control (MC) programmable logic controller (PLC), which serves as a logic-processing interface between the NB control computers and system hardware, was modified to improve the availability of NB heating of DIII-D plasmas in the event that one or more individual beam systems suddenly become unavailable while preparing for a tokamak experimental shot sequence. An upgraded computer platform was adopted for the NB control system operator interface and new graphical user interface pages were developed to more efficiently display system status data. A failure mode of the armor tile infrared thermometers (pyrometers), which serve to terminate beam pulsing if beam shine-through overheats wall thermal shielding inside the DIII-D tokamak, was characterized such that impending failures can be detected and repairs effected to mitigate beam system down-time. A scheme for remote testing of the pyrometers was also developed. The hardware that controls gas flow to the beamline neutralizer cells was upgraded to reduce susceptibility to electromagnetic interference (EMI), and interlocking was provided to terminate beam pulsing in the event of insufficient neutralizer gas flow, thereby preventing overheating of beamline internal components from excessive residual energetic ions. Ion source gas flow monitoring was also incorporated to aid in troubleshooting while conditioning beams. Finally, the beam pulse modulation system was upgraded to provide modulation while firing beam pulses into the calorimeters, allowing operators to optimize modulated beams before injecting them into the DIII-D tokamak. Motivation, implementation, and results of these improvements are presented.
\end{abstract}

\section{INTRODUCTION}

The DIII-D NB I\&C system, described in [1], comprises many electrical and electronic subsystems, which together control and monitor the NB ancillary systems, provide interlocking for safety and system integrity, and perform data aquisition. While the system has performed well for many years, recent upgrades have been incorporated to enhance function and reliability. Some obsolete equipment was replaced because it was no longer cost-effective to maintain, or because current technology made available much needed improved performance. Other equipment was modified to meet the changing needs of the DIII-D experimental program. Experience demonstrated that more data aquisition, control, or interlocking capability would provide improved protection of NB system components. It also became evident that integrity and electrical noise immunity of existing systems needed to be improved to keep the systems operating properly at present DIII-D experiment power and energy levels. The upgrades addressing these issues are discussed below.

\section{OPERATOR INTERFACE FOR NEW NB COMPUTERS}

The NB computers, which provided control and data acquisition functions for over 15 years, were recently replaced with more modern systems, as described in [2]. As part of this upgrade, the original operator "mode control panels" [3], which contained push-buttons, switches, and indicator lamps, were replaced by "virtual" control panels on the operators' console screens, or graphical user interfaces (GUI's). All previous control panel functions can now be performed by clicking a mouse cursor or observing color changes in appropriate screen fields.

Eliminating the physical control panel required reconfiguration of the relevant input/output signals (L/O). All signals that originated from switches and push-buttons on the control panel now originate from the operator's GUI. Rather than being fed into the control computer (via CAMAC interface digital input channels), some of these signals must now be sent out of the computer to communicate with other hardware. Likewise, external signals that once energized control panel indicator lamps are now fed into the computer for the GUI to properly display the indications. Signal paths strictly between components of the physical control panel and the computer were eliminated, since software and the GUI now handle these functions.

\footnotetext{
*Work supported by U.S. Department of Energy under Contract No. DE-AC03-89ER51114.
} 
The necessary wiring changes were made and $\mathrm{I} / \mathrm{O}$ channels were reconfigured. As a result, four physical control panels (one for each pair of neutral beams) were eliminated, along with their associated mechanical maintenance requirements. The eight DIII-D neutral beams are now operated from two computer terminal consoles, each comprised of a keyboard, a mouse, and three monitor displays.

\section{MODE CONTROL PROGRAM}

The NB Mode Control System, originally described in [3], is centered around a programmable logic controller, which serves as a logic-processing interface between the NB computers and system hardware. The program in the PLC synchronizes and coordinates the arming and firing of the neutral beams, based on which beams are to be utilized and in which operating mode.

For various reasons (power supply problems, lost interlocks, etc.), any of the neutral beams can become unavailable for injection during a get-ready/fire sequence. The philosophy utilized in the original design of the MC program dictated that neutral beams could be injected into the tokamak only if all the beams requested for a particular DIII-D experimental shot are available for that shot. If one or more beams did not complete their getready sequence, the program would not indicate that beams were ready for the experiment. It would then be necessary to manually initiate an abort for the errant beam systems. The abort cycle would further delay the experiment one to two minutes, before the program indicated that the remaining beams were ready. Likewise, if all requested beams successfully completed their respective get-ready cycles, and then one or more beams suddenly became unavailable, the program would again indicate that beams were not ready for the experiment, until the failed systems were aborted, and their abort cycles completed. This latter situation often occurred during the final moments before commencement of the DIII-D firing sequence, when it was nearly impossible to abort those beam systems in time for the DII-D shot. As a result, the shot would fire with no beams, a situation which usually constituted a worthless experimental shot.

It was eventually decided that it was better to fire a DIII-D shot with fewer beams than requested, than to fire the shot with no beams, or to have to abort the DIII-D shot. In addition, aborted beam systems should leave the remaining requested beams immediately available for the upcoming DIII-D shot. To meet these requirements, the MC PLC program was modified. The resulting logic now provides for the following:

(1) If one or more requested beam systems fails to get ready for an experimental shot, beams are indicated as not ready for the shot. This alerts scientists to the fact that they cannot get the beams they want for that shot.

(2) If a manual abort is initiated for the non-ready beams in (1) above, the remaining beam systems will immediately register as being ready for the shot. This provides scientists with the option of firing the DIII-D shot with only the remaining beams injecting, if deemed worthwhile. Abort cycle delays are eliminated. The scientists may always elect to abort the DIII-D shot and wait until the beam system problems can be rectified.

(3) If all requested beam systems successfully get ready for a shot, beams will register as ready. If one or more beam systems subsequently become unavailable (before the shot is fired), beams will continue to register as ready, and the remaining beams will fire as requested during the shot. This guarantees that some beams will still inject, even if others drop out during the last moments before the shot commences (when it is too late to respond to the drop-out by aborting the offending systems). Neutral beam operators are alerted any time a beam system drops out, and the scientific value of the DIII-D shot with the remaining beams can be assessed after the fact.

As a result of this upgrade to the MC PLC program, more successful DIII-D experimental shots are being fired. Shots with a total loss of beam heating have been minimized.

\section{CONTROL SYSTEM OPERATOR INTERFACE}

The NB Control System Operator Interface (Workstation) is a centralized graphical user interface for the NB I \& C system. The Workstation has been improved significantly since first being described in [4]. At that time the system primarily monitored the status of various interlocks, permits, valves, switches, etc. in the I \& C system. Display screens, which mimicked indicator panels in remote areas of the DIII-D facility, could be called up on the monitor, and "virtual" lamps on screens echoed the statuses of actual lamps on respective panels.

Over the last few years, the format of the screens was changed and control functions were added. It was decided that, for example, rather than having four separate beamline cooling water status screens, one for each beamline, it was expedient to combine the information into a single status screen. A glance at an array of indicators would quickly inform the operator which water channel, on which beamline, had a problem. Subsystem control screens, on the other hand, were developed individually for each beamline. These screens typically display a schematic of each subsystem. The $330^{\circ}$ beamline 
vacuum page, for example, displays the configuration of the various valves, pumps, pipes and gauges of that beamline's vacuum system. Object colors represent the status of various components (e.g. green valves are open, red valves are closed). In addition, clicking the mouse cursor on certain buttons in the diagram operates the components associated with those buttons. Valves can be opened and closed, gauges can be turned on and off, etc. The status of various components can be observed in quasi-real-time.

The various subsystem screens are all tied together through a "main" status screen. This screen displays the alarm statuses of all subsystems, for all four beamlines. Clicking the mouse cursor on a particular indicator immediately calls up the appropriate screen. In addition, all screens contain a set of screen call-up buttons, allowing the operator complete flexibility in jumping from one screen to another.

As functionality was added to the Workstation, the database grew, and performance degraded (screen changes and status updates took longer). It eventually became necessary to consider a new hardware platform for the Workstation. Originally comprised of a $33 \mathrm{MHZ}$ IBM compatible 486 computer with a 16 bit SVGA graphics adapter, the system was upgraded to a $100 \mathrm{MHZ}$ system with a 32 bit VL-Bus graphics adapter, resulting in dramatically improved performance. The Workstation is now in continuous use by the NB group, and is relied upon for set-up and shut-down functions, remote control of most subsystems, and as a first source of information and control when subsystem malfunctions occur.

\section{ARMOR TILE INFRARED THERMOMETERS}

The armor tile infrared thermometers (pyrometers) optically measure temperature of the thermal shielding armor tiles on the wall inside the DIII-D vacuum vessel in line with the neutral beam paths. If beam shine-through overheats these tiles, the pyrometers trip interlocks to terminate pulsing of the appropriate beams. Over the last two years, the failure rate of the pyrometers has noticeably increased. Because some recent DIII-D experiments have produced record neutron numbers, and because failed pyrometer sense heads removed from the DIII-D machine enclosure typically exhibit radioactivation as high as 400 to 500 counts per minute, it is suspected that neutron damage is responsible for these failures.

Because radioactive pyrometers could not be shipped to the manufacturer for repairs, on-site troubleshooting was necessary. A degraded internal electronic component was identified in each of the failed pyrometer sense heads. New components were obtained from the manufacturer and installed in the pyrometer sense heads. The units were recalibrated and returned to service.
An interesting characteristic of the failure mode was noticed. As degradation of the internal component progressed, the temperature displayed when the pyrometer's self-calibration feature was activated would suddenly appear significantly lower. (The unit's self calibration control could be adjusted to bring the system barely back into proper calibration.) Then, with further degradation, self-calibration would suddenly display no temperature reading. This step-wise failure mechanism became an important indicator of impending total pyrometer failure, allowing continuing use of the system after the first "warning". When a large interval signal drop is noticed upon self-calibration testing of a pyrometer, the unit can be self-recalibrated in a matter of minutes, allowing neutral beam operations to continue without interruption. A note is made that the pyrometer's performance is degrading, and the unit can be replaced with a spare in about two hours that evening. The failed unit can then be repaired, fully recalibrated, and returned to the stock of usable spares in about a day's time.

Because of this early failure detection capability, downtime of the neutral beam systems due to unexpected pyrometer failures is minimized. In addition, now that the likely failure mode is understood, spare parts can be stocked, and failing units can be quickly repaired on-site at the DIII-D facility, maintaining the necessary supply of spare pyrometers. Interfacing is also being developed which would allow the NB operators to perform calibration checks of the pyrometer systems from their control consoles. This would encourage more frequent checking of the pyrometers, resulting in earlier detection of impending failures.

The above procedures are being utilized as an interim measure until this particular type of pyrometer failure can be prevented. A new pyrometer system is being assessed, in which fiber optics would allow the sense head to be moved up to twenty feet further away from the DIII-D tokamak, thereby reducing neutron exposure. Also under consideration is the replacement of sensitive electronic components with "radiation-hardened" versions.

\section{GAS FLOW CONTROL AND MONITORING}

During beam pulsing, deuterium gas is puffed into the beamline neutralizer cell to optimize neutralization of the accelerated ion beam. A commercially available mass flow controller (MFC) regulates the gas flow and provides a signal representative of the flow rate. The flow rate setpoint is entered by the NB operator at the control console, and feeds out of a CAMAC module in the DII-D machine enclosure, where it connects to the MFC. The flow rate signal from the MFC is digitized in another CAMAC module, and the resulting waveform is displayed at the operator's console. 
A relay, operated by a timed gate, originally switched the setpoint signal into and out of the MFC input during a beam pulse sequence. Under certain conditions, erratic operation of the MFC was observed, and troubleshooting indicated that stray magnetic fields in the machine enclosure were affecting the relay.

A new gas pulse control interface was developed to address this problem. The interface utilizes solid-state analog switches rather than electromagnetic relays. A fiberoptic-isolated timed gate now activates the analog switches to provide the MFC setpoint, and also operates the "valve off" bit of the MFC, providing redundant control of gas pulsing. Since this upgrade, the neutralizer gas pulse system has performed extremely well, exhibiting no susceptibility to EMI.

To provide additional protection against insufficient gas flow to the neutralizer cell, a "neutralizer gas interlock" was implemented. During beam injection, the interlock circuit monitors the gas flow signal produced by the MFC. If the flow rate does not reach a minimum threshold after a programmed amount of time, a fault is sensed. If the fault persists beyond another programmed delay, beam pulsing is terminated. The interlock successfully prevents overheating of beamline components from residual energetic ions in insufficiently neutralized beams.

Deuterium flow to each NB ion source is controlled by a manually adjustable orifice and an electrically operated solenoid valve. To verify proper gas flow during beam pulsing, MFC's utilized as flow meters (with disabled flow control mechanisms) were installed in the ground-level gas feeds to the ion sources. Gas flow signals are digitized and displayable at the operator's console. Monitoring ion source gas flow helps distinguish gas system problems from other operational problems when beams are not operating optimally.

\section{CALORIMETER-MODE BEAM PULSE MODULATION}

Beam pulse modulation, or chopping a long beam pulse into a series of repetitive shorter pulses, allows DIII-D scientists greater flexibility in controlling NB power injected into the tokamak plasma. Recent experiments have required higher modulation frequencies, and NB operators have been experiencing problems keeping beams "tuned" at these frequencies. To provide the operators more opportunity to optimize the modulated beams, the modulation system was modified to operate while firing beam pulses into the calorimeters, as well as when injecting into DIII-D plasmas.
With the original modulation system, scientists at a computer terminal program timing information which "chops" the beam pulse commands during a DIII-D plasma shot. To upgrade the system, additional control was added, programmable only by NB operators. This part of the system affects beams which are not in synchronization with DIII-D operation. As the result of the upgrade, NB operators can now tune and optimize modulated beams during conditioning, helping assure that these beams will be ready for experiments requiring modulated beams.

\section{SUMMARY}

Recent upgrades have improved the overall reliability and capability of the DIII-D neutral beam systems. Full interfacing to the new NB computers has provided beam operators complete operational control from their console terminals, utilizing modern computer hardware and software, and eliminating mechanical control panels. Upgrades to the mode control PLC program have minimized "wasted" DIII-D shots by allowing some beams to inject even when other requested beams suddenly become unavailable. The NB Workstation has been developed into a useful monitoring and control center, providing quick access to beam subsystem status information, saving time when subsystem manual control is necessary, and providing redundant data aquisition and control for the beam subsystems, should other control systems fail. Improved understanding of the armor tile pyrometers and a particular failure mode has resulted in early detection and quick response to such impending failures, minimizing associated down-time. Upgrades to gas systems have improved the reliability of gas pulse control, have increased protection to beamline components, and have enhanced gas flow monitoring capability. Finally, combined with these improvements, the provision of beam modulation in calorimeter conditioning mode has increased the readiness of beams for particular experiments, making neutral beams an even more valuable asset to the DIII-D program.

\section{REFERENCES}

[1] J. C. Kohli, D.D. Drobnis, V.P. Elischer, R. Kilgore, D. Uber, "Instrumentation and control of the Doublet III neutral beam injection system," in Proc. 8th Symp. on Eng. Problems of Fusion Research, (1979) pp. 924-928.

[2] J. Phillips, et al., "Operational advances using the new DIII-D neutral beam computer systems", this conference.

[3] J. C. Kohli, et al., "Neutral beam mode control", in Proc. 9 th Symp. on Eng. Problems of Fusion Research, (1981) pp. 2058-2061.

[4] J.J. Harris and G.L. Campbell, "DIII-D neutral beam control system operator interface," in Proc. 15th Symp. on Fus. Eng. (1993) pp. 554-557. 